DOI: $10.2478 /$ ace-2014-0029

\title{
VIBRATIONS AND STABILITY OF BERNOULLI-EULER AND TIMOSHENKO BEAMS ON TWO-PARAMETER ELASTIC FOUNDATION
}

\author{
P. OBARA ${ }^{1}$
}

\begin{abstract}
The vibration and stability analysis of uniform beams supported on two-parameter elastic foundation are performed. The second foundation parameter is a function of the total rotation of the beam. The effects of axial force, foundation stiffness parameters, transverse shear deformation and rotatory inertia are incorporated into the accurate vibration analysis. The work shows very important question of relationships between the parameters describing the beam vibration, the compressive force and the foundation parameters. For the free supported beam, the exact formulas for the natural vibration frequencies, the critical forces and the formula defining the relationship between the vibration frequency and the compressive forces are derived. For other conditions of the beam support conditional equations were received. These equations determine the dependence of the frequency of vibration of the compressive force for the assumed parameters of elastic foundation and the slenderness of the beam.
\end{abstract}

Keywords: Bernoulli-Euler beam, Timoshenko beam, elastic foundation, stability, vibration

\section{INTRODUCTION}

The stability and dynamic analyses of beams or beam-columns belong to the classical problems of the structural mechanics [the base items 1-3]. These analyses are carried out by using Bernoulli-Euler beam theory for the case of slender beams or Timoshenko theory for stocky beams (the beams with small length-to-depth ratio). In the first one, straight lines or planes normal to the neutral beam axis remain straight and normal after deformation, in the second one, the influence of transverse shear deformation and rotatory inertia is considered $[4,5]$. These models fairly realistically describe the behavior of the beams, but the description of subsoil and its interaction with a beam resting on it is not easy.

The soil-structure interaction problems occupy an important place in many fields of structural and foundation engineering, e.g. in the analysis of building, geotechnical, highway, and railroad structures, submerged pipes, etc. For over a century, various physical and mathematical foundation models, approximating the real behavior, were formulated. These foundations are characterized by one, two or more parameters $[6,7]$.

1 Kielce University of Technology, Faculty of Civil and Environmental Engineering, Al. 1000-lecia PP 7, 25-314 Kielce, Poland, e-mail: paula@tu.kielce.pl 
The oldest, most frequently used model, was formulated in 1867 by Winkler [8]. In that model, the beam-supporting soil is modeled as a series of closely spaced, mutually independent, linear elastic vertical springs which, evidently, provide resistance in direct proportion to the deflection of the beam. In the Winkler model, the properties of the soil are described by only one parameter, which represents the stiffness of the vertical spring. Although the model represents the simplest form of elastic foundation, in most practical applications, it is used to model soil behavior. Timoshenko and Gere [2] proposed a solution for simply supported uniform beams resting on the Winkler type foundation. Free vibration and stability analysis of beams resting on the Winkler foundation was studied in Refs [9-12]. The post-critical loads for Euler and Beck columns resting on the elastic foundation were presented in Ref. [13]. In Ref. [14], the thermal buckling and post-buckling of a pinned-fixed beam was investigated. The vibration and stability analyses of an infinite Bernoulli-Euler beam and an infinite shear beam-column resting on the Winkler-type elastic foundation, by using a Fourier transform, were performed by Kim $[15,16]$. In those papers, the analyses were carried out for the system subjected to a static axial force and a moving load with either constant or harmonic amplitude variations. In Ref. [17], a beam on equidistant elastic supports was considered as a beam on the elastic foundation in static and free vibration problems.

Many researchers have aimed to generalize and improve the Winkler model frequently adopted to solve soil-structure interaction problems. More realistic hypothesis is considered in the elastic foundation model with two parameters. The most commonly used foundation models include the following: the Pasternak model [18], the Filonienko-Borodich model [19], and the Vlasov-Leontiev model [20]. In those models, the first parameter represents the stiffness of the vertical spring, as in the Winkler model, whereas the second parameter is introduced to account for the coupling effect of the linear springs. In the first case, the second parameter can be considered as the shear stiffness of a shear layer and in the second - as the tension in an elastic membrane connecting the top ends of the Winkler springs. The last model consists of an elastic layer resting on the non-deformable base. The analysis using Vlasov model was examined in Refs [21-23].

The analysis of a beam resting on two-parametrical elastic foundation has been conducted by many authors [24-33]. A majority of them employed a finite element formulation to perform analyses. For example, Naidu and Rao were concerned with the stability analysis [24] and the vibration behavior [25] of the Bernoulli-Euler beam resting on the modified Pasternak model. The effect of the elastic foundation on buckling loads for various end boundaries was examined in those papers. The finite element technique for determining the vibration characteristics of a Bernoulli-Euler and Timoshenko beam was also used by Yokoyama [26]. In that work, the effect of axial force, foundation stiffness parameters, transverse shear deformation and rotatory inertia were incorporated into the finite element model. In Ref. [27] approximate explicit formulas for the fundamental natural vibration frequency of Timoshenko beams mounted on the Pasternak foundation were derived. The dynamic stiffness matrix and the load vector of the Timoshenko beam-column resting on the two-parameter elastic foundation 
with generalized end condition were presented in Ref. [28]. The static, dynamic and stability behavior of framed structures made of beam-columns were analyzed in that paper. Free vibration frequencies of Timoshenko beams on two-parameter elastic foundation were examined by Rosa [29] for two different models. In the first model, the second foundation parameter is assumed to be a function of the flexural rotation, whereas in the second model, it is assumed to be a function of the global cross-section rotation. Studies of analytical considerations of stability and vibration are generally not readily available in the literature.

The purpose of this article is to present a general formulation for the vibration and stability problems of beams with various boundary conditions, supported on two-parameter elastic foundation. The second foundation parameter is a function of the total rotation of the beam, like in Ref. [29]. The effects of the shear deformation, rotatory inertia and the foundation parameters on the frequency and critical loads of the beam are discussed in detail. Wherein, it should be stressed that the relationship between the parameters describing the beam vibration, the compressive force and the foundation parameters will be considered.

\section{THEORY AND FORMULATION}

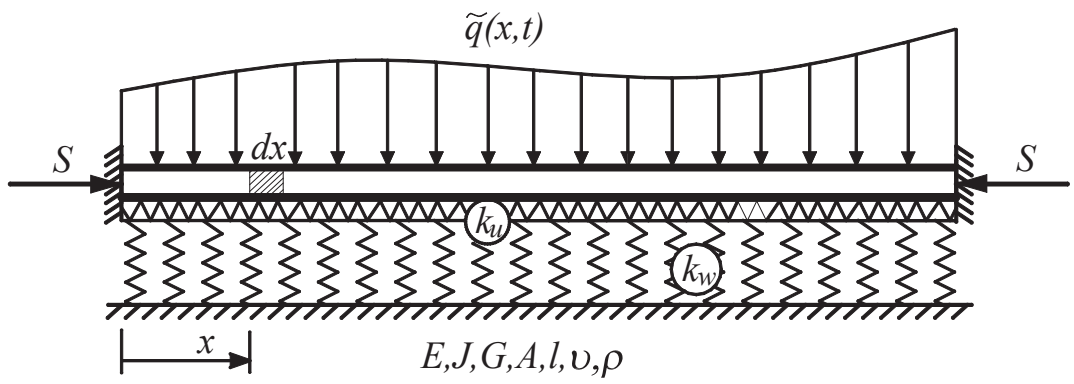

Fig. 1. Model of a beam with an axial force resting on a two-parameter elastic foundation.

Consider a beam of initial length $l$, axially compressed with force $S$, resting on the elastic foundation, as shown in Figure 1. In the presented formulation, it is assumed that: (1) the beam is made of an isotropic homogenous linear elastic material with the Young's modulus $E$, the shear modulus $G$, the Poisson's ratio $v$; (2) the transverse cross-section of the beam is doubly symmetric with respect to the height of beam $h$, the area $A$, the moment of inertia $J$; (3) the beam has uniform mass density $\rho$ per unit length; (4) the shear coefficient depending on the shape of cross-section $\kappa$ is taken into account; (5) the central axis is a straight line; (6) the vibration amplitudes of the beam are sufficiently small; (7) the damping of the foundation are negligible; (8) the bonding between the beam and foundation is perfect. 
The analysis is made for Timoshenko beam model. As a result, the effects of transverse shear deformation and rotatory inertia are taken into account. The cross-section initially normal to the neutral axis of the beam remains plane, but no longer normal to that axis in bending. The slope of the deflection curve $\widetilde{w}^{I}(x, t)$ depends on the rotation of the beam cross-section, $\widetilde{\varphi}(x, t)$, and additionally, on the average shear deformation angle, $\kappa \widetilde{\psi}(x, t)$ :

$$
\frac{\partial \widetilde{w}(x, t)}{\partial x}=\widetilde{\varphi}(x, t)+\kappa \widetilde{\psi}(x, t)
$$

The effect of the rotatory inertia is expressed by:

$$
\widetilde{M}^{B}(x, t)=\rho J \frac{\partial^{2} \widetilde{\varphi}(x, t)}{\partial t^{2}} d x
$$

The section forces can be expressed by the deflection and rotation function as follows:

$$
\begin{gathered}
\widetilde{T}(x, t)=G A \widetilde{\psi}(x, t)=\frac{G A}{\kappa}\left(\frac{\partial \widetilde{w}(x, t)}{\partial x}-\widetilde{\varphi}(x, t)\right) \\
\widetilde{M}(x, t)=-E J \frac{\partial \widetilde{\varphi}(x, t)}{\partial x}
\end{gathered}
$$

The elastic foundation is idealized as a constant two-parameter model characterized by two moduli, i.e. the vertical foundation modulus $k_{w}$ (the Winkler parameter) and the horizontal foundation modulus $k_{u}$. In the case $k_{u}=0$, this model is reduced to the usual Winkler model. The foundation response is taken into account as the vertical ground reaction, proportional to the vertical displacement $\widetilde{w}(x, t)$ :

$$
\widetilde{q}_{S}(x, t)=k_{w} \widetilde{w}(x, t)
$$

and the horizontal reaction proportional to the horizontal displacement of the extreme fibres of the beam $\widetilde{u}(x, t)$. This effect is taken into account in the form of the bending moment:

$$
\widetilde{m}_{S}(x, t)=k_{\varphi} \widetilde{\varphi}(x, t)
$$

where $k_{\varphi}=k_{u} h^{2} / 4$ 


\subsection{THE DIFFERENTIAL EQUATION DERIVATION}

The dynamic equilibrium on the differential element of the beam is shown in Figure 2. Assuming a small curvature in the current configuration $(\cos (\partial \widetilde{w} / \partial x) \cong 1$, $\sin (\partial \widetilde{w} / \partial x) \cong \partial \widetilde{w} / \partial x)$, the transverse and rotational equilibrium equations are:

$$
\left\{\begin{array}{l}
\frac{\partial \widetilde{T}}{\partial x}-S \frac{\partial^{2} \widetilde{w}}{\partial x^{2}}-A \rho \frac{\partial^{2} \widetilde{w}}{\partial t^{2}}-k_{w} \widetilde{w}+\widetilde{q}=0 \\
\frac{\partial \widetilde{M}}{\partial x}-\widetilde{T}+J \rho \frac{\partial^{2} \widetilde{\varphi}}{\partial t^{2}}+k_{\varphi} \widetilde{\varphi}-\widetilde{m}=0 .
\end{array}\right.
$$

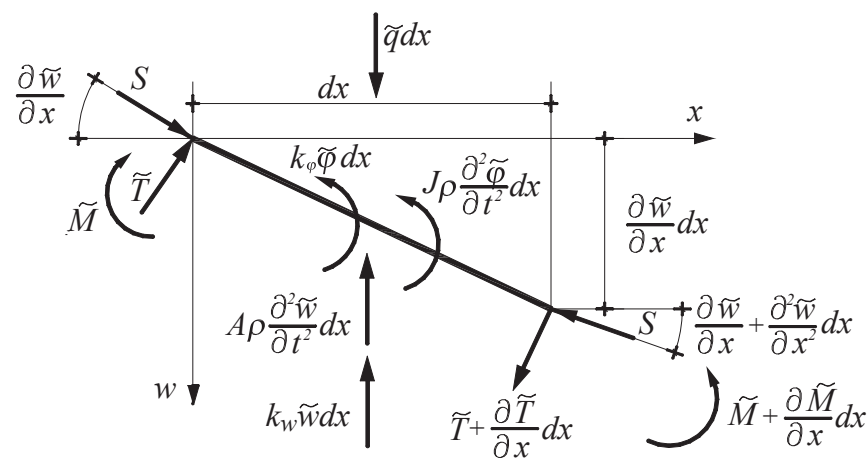

Fig. 2. Forces, moments and deformations of the differential element

Substituting Eqs. (2.3) and (2.4) into (2.7) results in

$$
\left\{\begin{array}{l}
\frac{G A}{\kappa}\left(\frac{\partial^{2} \widetilde{w}}{\partial x^{2}}-\frac{\partial \widetilde{\varphi}}{\partial x}\right)-S \frac{\partial^{2} \widetilde{w}}{\partial x^{2}}-A \rho \frac{\partial^{2} \widetilde{w}}{\partial t^{2}}-k_{w} \widetilde{w}+\widetilde{q}=0 \\
-E J \frac{\partial^{2} \widetilde{\varphi}}{\partial x^{2}}-\frac{G A}{\kappa}\left(\frac{\partial \widetilde{w}}{\partial x}-\widetilde{\varphi}\right)+J \rho \frac{\partial^{2} \widetilde{\varphi}}{\partial t^{2}}+k_{\varphi} \widetilde{\varphi}=0 .
\end{array}\right.
$$

From now on, the free harmonic vibrations of the beam will be considered $(\widetilde{q}=0)$. Following the introduction of the non-dimensional coordinate along the axis of the beam $\xi=x / l ; \xi \in\langle 0,1\rangle$ and parameters describing the beam:

$$
\sigma^{2}=\frac{S l^{2}}{E J}, \quad \varepsilon^{2}=\frac{1}{1-\sigma^{2} \zeta}, n=\frac{G}{\kappa E}, \zeta=\frac{\kappa E J}{G A l^{2}}, \mu=A \rho, \chi^{4}=\frac{\mu l^{4}}{E J}
$$

and the foundation: 


$$
\lambda_{w}^{4}=\frac{k_{w} l^{4}}{E J}, \lambda_{\varphi}^{4}=\frac{k_{\varphi} l^{2}}{E J}
$$

equations (2.8) can be written in the form:

$$
\begin{gathered}
\widetilde{w}^{I V}+\left(\sigma^{2} \varepsilon^{2}-\lambda_{w}^{4} \varepsilon^{2} \zeta-\lambda_{\varphi}^{4}\right) \widetilde{w}^{I I}+\lambda_{w}^{4} \varepsilon^{2}\left(1+\lambda_{\varphi}^{4} \zeta\right) \widetilde{w}+ \\
-\chi^{4} \zeta\left(\varepsilon^{2}+n\right) \ddot{\tilde{w}}^{I}+\chi^{4} \varepsilon^{2}\left(1+\lambda_{\varphi}^{4} \zeta+\lambda_{w}^{4} \zeta^{2} n\right) \ddot{\tilde{w}}+\chi^{8} \varepsilon^{2} \zeta^{2} n \widetilde{\widetilde{w}}=0 \\
\widetilde{\varphi}\left(1+\lambda_{\varphi}^{4} \zeta\right)+\chi^{4} \zeta^{2} n \ddot{\widetilde{\varphi}}=\frac{1}{l}\left[\left(1-\sigma^{2} \zeta\right) \zeta \widetilde{w}^{I I}-\chi^{4} \zeta^{2} \ddot{\widetilde{w}}^{I}+\left(1-\lambda_{w}^{4} \zeta^{2}\right) \widetilde{\widetilde{w}}^{I}\right]
\end{gathered}
$$

Applying the separation of variables to the functions $\widetilde{w}(\xi, t)$ and $\widetilde{\varphi}(\xi, t)$ :

$$
\widetilde{w}(\xi, t)=w(\xi) e^{i \omega t}, \quad \widetilde{\varphi}(\xi, t)=\varphi(\xi) e^{i \omega t}
$$

where $\omega$ is the circular vibration frequency, and substituting into (2.11), the differential equation of the motion of the harmonic amplitudes is obtained:

$$
w^{I V}(\xi)+2 X w^{I I}(\xi)+Y w(\xi)=0
$$

where:

$$
\begin{gathered}
2 X=\varepsilon^{2}\left(\sigma^{2}-\lambda_{w}^{4} \zeta\right)-\lambda_{\varphi}^{4}+\lambda^{4} \zeta\left(\varepsilon^{2}+n\right), Y=\varepsilon^{2}\left(\lambda_{w}^{4}-\lambda^{4}\right)\left(1+\lambda_{\varphi}^{4} \zeta-\lambda^{4} \zeta^{2} n\right), \\
\lambda^{4}=\frac{\mu \omega^{2} l^{4}}{E J}
\end{gathered}
$$

After substituting Eqs. (2.13) into (2.12), the total rotation of the beam cross-section has following form:

$$
\varphi(\xi)=\frac{1}{l}\left[e w^{I I I}(\xi)+d w^{I}(\xi)\right]
$$

where:

$$
e=\frac{\left(1-\sigma^{2} \zeta\right) \zeta}{1+\lambda_{\varphi}^{4} \zeta-\lambda^{4} \zeta^{2} n}, \quad d=\frac{1+\lambda^{4} \zeta^{2}-\lambda_{w}^{4} \zeta^{2}}{1+\lambda_{\varphi}^{4} \zeta-\lambda^{4} \zeta^{2} n}
$$


A number of specific cases of equations (2.14) and (2.16) can be distinguished. If $\omega=0$, the equations describe the stability of Timoshenko beam. If $k_{w}=k_{u}=0$ $\left(\lambda_{w}^{4}=0, \lambda_{\varphi}^{4}=0\right)$ the equations describe free vibrations or the stability of Timoshenko beam with no elastic foundations to rest on. If $\zeta=0(\varepsilon=0),(2.14)$ and (2.16) become the equations for Bernoulli-Euler beam and then $2 X=\sigma^{2}-\lambda_{\varphi}^{4}, Y=\lambda_{w}^{4}-\lambda^{4}$, and $\varphi(\xi)=1 / l w^{I}(\xi)$.

The solution of homogeneous differential equation (2.14) is the function:

$$
w(\xi)=C_{1} e^{k_{1} \xi}+C_{2} e^{k_{2} \xi}+C_{3} e^{k_{3} \xi}+C_{4} e^{k_{4} \xi}
$$

where the coefficients $k_{i},(i=1,2,3,4)$ are the roots of the characteristic equation:

$$
k_{1,2}= \pm \sqrt{k_{1}^{2}}, \quad k_{3,4}= \pm \sqrt{k_{2}^{2}}
$$

where:

$$
k_{1}^{2}=-X+\sqrt{X^{2}-Y}, \quad k_{2}^{2}=-X-\sqrt{X^{2}-Y} .
$$

The roots (2.19) depend on the relationships between the coefficients $2 X$ and $Y$.

\subsection{The ANALYSis OF THE RESULting SOLUtion}

In the orthogonal coordinate system $2 X, Y$ (Figure 3), the parabola $Y=X^{2}$ and the $2 X$-axis split up the $2 X, Y$-plane into four regions, in which the following relationships between coefficients $2 X$ and $Y$ hold:

$$
\text { I: } 2 X>0 \wedge 0<Y<X^{2} \text {, II: } Y>X^{2} \text {, II: } 2 X<0 \wedge 0<Y<X^{2} \text {, IV: } Y<0 \text {. }
$$

On the basis of the relationships (2.21) taking into account that the foundation parameters $k_{u}$ and $k_{w}$ and the shear deformation parameter $\zeta$ are non-negative values, we can conclude that:

- if the stability analysis of Timoshenko and Bernoulli-Euler beam on one or twoparameter elastic foundation is carried out, the coefficients $2 \mathrm{X}$ and $\mathrm{Y}$ satisfy the conditions for the region I,

- if the static analysis of beams on elastic foundations is performed, the conditions characterizing the region II occur,

- if the tensile forces affect the beams, the coefficients $2 \mathrm{X}$ and $\mathrm{Y}$ satisfy the inequalities characterizing the region III,

- the phenomenon of free vibration or vibration of the tensile or compressive loads of the beam resting on elastic foundations is described by the coefficients $2 \mathrm{X}$ and $\mathrm{Y}$ satisfying the inequalities characterizing the region IV, 
- the case, for which $2 X=0$ and $Y=0$ (the origin $2 X Y$ ), describes a static analysis of beams with no elastic foundations to rest on.

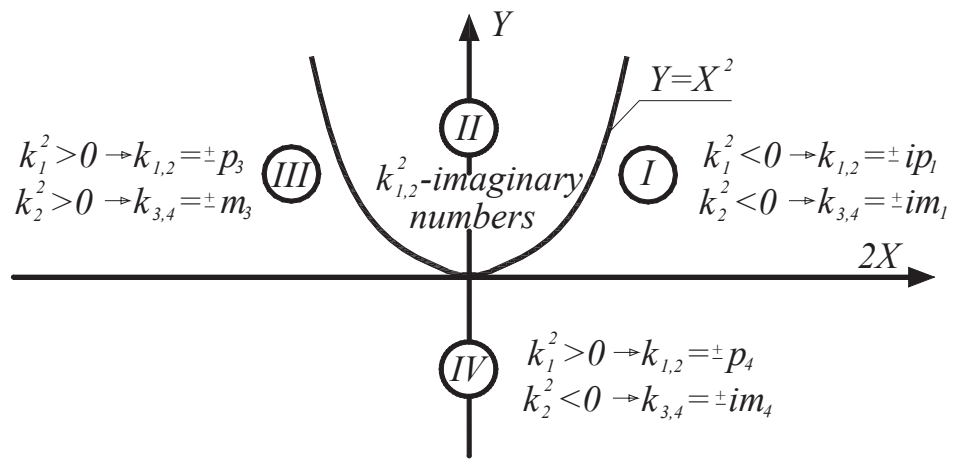

Fig. 3. The division of $2 X, Y$ plane into four areas

Figure 3 shows geometrical picture of all the possible cases of magnitudes and the relationships between the coefficients $2 X$ and $Y$ appearing in the differential equation (2.14). At the same time, the relationships (2.21) enable us to find out whether the parameters $k_{i}(2.19)$ are real, imaginary or complex numbers. Thus, a form of general integral (2.18) of the differential equation (2.14) depends upon which of the relationships (2.21) is satisfied by the coefficients $2 X$ and $Y$.

\subsubsection{The SOLUTION OF EQUATION (2.14) IN REGIONS I, III AND IV}

If the coefficients $2 X$ and $Y$ take their values from the first region $k_{1}^{2}$ and $k_{2}^{2}$ are negative real numbers, $k_{1,2}$ and $k_{3,4}$ have imaginary values:

$$
k_{1,2}= \pm i p_{1}, \quad k_{3,4}= \pm i m_{1}
$$

where:

$$
p_{1}=\sqrt{X-\sqrt{X^{2}-Y}}, \quad m_{1}=\sqrt{X+\sqrt{X^{2}-Y}}
$$

In the third region $k_{1}^{2}$ and $k_{2}{ }^{2}$ are positive real numbers, so $k_{1,2}$ and $k_{3,4}$ are actual values:

$$
k_{1,2}= \pm p_{3}, \quad k_{3,4}= \pm m_{3}
$$

where: 


$$
p_{3}=\sqrt{-X+\sqrt{X^{2}-Y}}, \quad m_{3}=\sqrt{-X-\sqrt{X^{2}-Y}}
$$

In the fourth region, $k_{1}^{2}$ is a positive, but $k_{2}^{2}$-a negative number, therefore the roots $k_{1,2}$ are real and $k_{3,4}$ are imaginary numbers:

$$
k_{1,2}= \pm p, \quad k_{3,4}= \pm i m
$$

where:

$$
p=\sqrt{\sqrt{X^{2}-Y}-X}, m=\sqrt{\sqrt{X^{2}-Y}+X} .
$$

If we consider the relationship between the parameters $p_{i}$ and $m_{i}\left(p_{i}=p, m_{1}=m, p_{3}=p\right.$, $\left.m_{3}=i n\right)$ and trigonometric dependencies:

$$
\cosh i z=\cos z, \quad \sinh i z=i \sin z
$$

the general integral (2.18) can be written finally in the form:

$$
w(\xi)=C_{1} \cosh p \xi+C_{2} \sinh p \xi+C_{3} \cos m \xi+C_{4} \sin m \xi
$$

The amplitude equation of the rotation of the beam cross-section is received by substituting derivatives of the function (2.29) to (2.16):

$$
\varphi(\xi)=\frac{1}{l}\left[C_{1} A \sinh p \xi+C_{2} A \cosh p \xi+C_{3} B \sin m \xi-C_{4} B \cos m \xi\right]
$$

where:

$$
A=e p^{3}+d p, \quad B=e m^{3}-d m .
$$

\subsubsection{The SOlution OF EQUATION (2.14) IN REGION II}

If the coefficients $2 X$ and $Y$ take their values from the second region $k_{1}^{2}$ and $k_{2}^{2}$ are complex numbers, $k_{1,2}$ and $k_{3,4}$ are complex numbers, too:

$$
k_{1,2,3,4}= \pm \sqrt[4]{Y}\left(\cos \frac{1}{2} v \pm i \sin \frac{1}{2} v\right) ; \cos v=-\frac{X}{\sqrt{Y}}
$$

where $v$ is an amplitude of a complex number.

The general integral (2.18) for this cases can be expressed as: 


$$
\begin{aligned}
w(\xi)= & C_{1} \cosh p_{2} \xi \cos m_{2} \xi+C_{2} \cosh p_{2} \xi \sin m_{2} \xi+C_{3} \sinh p_{2} \xi \cos m_{2} \xi+ \\
& +C_{4} \sinh p_{2} \xi \sin m_{2} \xi
\end{aligned}
$$

where:

$$
p_{2}=\sqrt[4]{Y} \cos \frac{1}{2} v=\sqrt{\frac{\sqrt{Y}-X}{2}}, \quad m_{2}=\sqrt[4]{Y} \sin \frac{1}{2} v=\sqrt{\frac{\sqrt{Y}+X}{2}}
$$

and the amplitude equation of the rotation of the beam cross-section (2.16) may be written as:

$$
\begin{aligned}
l \varphi(\xi)= & C_{1}\left[E \cos m_{2} \xi \sinh p_{2} \xi+F \cosh p_{2} \xi \sin m_{2} \xi\right]+ \\
& +C_{2}\left[E \sin m_{2} \xi \sinh p_{2} \xi+F \cosh p_{2} \xi \cos m_{2} \xi\right]+ \\
& +C_{3}\left[E \cos m_{2} \xi \cosh p_{2} \xi+F \sinh p_{2} \xi \sin m_{2} \xi\right]+ \\
& +C_{4}\left[E \sin m_{2} \xi \cosh p_{2} \xi+F \sinh p_{2} \xi \cos m_{2} \xi\right]
\end{aligned}
$$

where:

$$
E=p_{2}\left(d+e\left(p_{2}^{2}-3 m_{2}^{2}\right)\right), \quad F=-m_{2}\left(d-e\left(m_{2}^{2}-3 p_{2}^{2}\right)\right) .
$$

\subsection{THE DETERMinATION OF THE EIGENVALUES}

Analyzing the differential equation (2.14), among the derived expansion functions, the expressions can be distinguished that describe the deflected function of the beam under free vibrations or under buckling. In both cases, it is the function (2.29). Four integration constants $C_{i}$ appearing in the (2.29) are to be calculated by imposing the boundary conditions. For the simply supported beam, boundary conditions:

$$
w(\xi=0)=0 ; \quad M(\xi=0)=0 ; \quad w(\xi=1)=0 ; \quad M(\xi=1)=0
$$

lead to the following homogeneous system of equations:

$$
\left[\begin{array}{cccc}
1 & 0 & 1 & 0 \\
A p & 0 & B m & 0 \\
\cosh p & \sinh p & \cos m & \sin m \\
A p \cosh p & A p \sinh p & B m \cos m & B m \sin m
\end{array}\right] *\left[\begin{array}{l}
C_{1} \\
C_{2} \\
C_{3} \\
C_{4}
\end{array}\right]=\left[\begin{array}{l}
0 \\
0 \\
0 \\
0
\end{array}\right]
$$


The condition, from which we determine the eigenvalues, is resetting the primary determinant of equations (2.38), which leads to the equation:

$$
-(B m-A p)^{2} \sin m \sinh p=0
$$

whose fulfillment is a condition of equilibrium bifurcation of the element under compression. The equation (2.39) has solutions for:

$$
m=k \pi ; \quad k=1,2,3 \ldots
$$

which, after using the formulas $(2.27)_{2},(2.15)$ leads to the dependence:

$$
\sigma=\sqrt{\frac{\lambda_{w}^{4}-\lambda^{4}}{k^{2} \pi^{2}}+\frac{\lambda_{\varphi}^{4}-\lambda^{4} \zeta n+k^{2} \pi^{2}}{1+\left(\lambda_{\varphi}^{4}-\lambda^{4} \zeta n+k^{2} \pi^{2}\right) \zeta}}
$$

If $\lambda=0$, we obtain the formula for the critical compressive force:

$$
S_{K R}=\left[\frac{\lambda_{w}^{4}}{k^{2} \pi^{2}}+\frac{\lambda_{\varphi}^{4}+k^{2} \pi^{2}}{1+\left(\lambda_{\varphi}^{4}+k^{2} \pi^{2}\right) \zeta}\right] \frac{E J}{l^{2}}
$$

and if $\sigma=0-$ two natural frequency bands are received:

$$
\omega_{1 k}=\sqrt{\frac{b-\sqrt{b^{2}-4 a c}}{2 a}} \sqrt{\frac{E J}{\mu l^{4}}} ; \quad \omega_{2 k}=\sqrt{\frac{b+\sqrt{b^{2}-4 a c}}{2 a}} \sqrt{\frac{E J}{\mu l^{4}}}
$$

where:

$$
\begin{aligned}
& a=n \zeta^{2}, \quad b=1+\left[\lambda_{\varphi}^{4}+k^{2} \pi^{2}+n\left(\lambda_{w}^{4} \zeta+k^{2} \pi^{2}\right)\right] \zeta \\
& c=\left(\lambda_{\varphi}^{4}+k^{2} \pi^{2}\right)\left(\lambda_{w}^{4} \zeta+k^{2} \pi^{2}\right)+\lambda_{w}^{4} .
\end{aligned}
$$

If we do not take into account the effect of rotational inertia, we get the formula for one natural frequency band:

$$
\omega_{1 k}=\sqrt{\frac{\left(\lambda_{\varphi}^{4}+k^{2} \pi^{2}\right)\left(\lambda_{w}^{4} \zeta+k^{2} \pi^{2}\right)+\lambda_{w}^{4}}{1+\left(\lambda_{\varphi}^{4}+k^{2} \pi^{2}\right) \zeta}} \sqrt{\frac{E J}{\mu l^{4}}} .
$$


A number of specific cases of the formulas (2.42) and (2.45) can be distinguished. If $k_{w}=k_{u}=0 \quad\left(\lambda_{w}^{4}=0, \quad \lambda_{\varphi}^{4}=0\right)$ the equations describe the critical compressive force and the natural frequency of Timoshenko beam with no elastic foundations to rest on. If $\zeta=0(\varepsilon=1),(2.42)$ and (2.45) become the formulas of eigenvalues for Bernoulli-Euler beam (Table 1$)$.

Table 1

The formulas for the critical compressive force $S_{K R}$ and the natural frequency $\omega$ for Bernoulli-Euler and Timoshenko simply supported beam

\begin{tabular}{|c|c|c|c|}
\hline & Bernoulli-Euler & \multicolumn{2}{|c|}{ Timoshenko } \\
\cline { 2 - 4 } & beam with elastic foundation to rest on & \multicolumn{2}{|c|}{ beam with no elastic foundation to rest on } \\
\hline$S_{K R}\left[E J / l^{2}\right]$ & $\frac{k^{4} \pi^{4}+k^{2} \pi^{2} \lambda_{\varphi}^{4}+\lambda_{w}^{4}}{k^{2} \pi^{2}}$ & $k^{2} \pi^{2}$ & $\frac{k^{2} \pi^{2}}{1+k^{2} \pi^{2} \zeta}$ \\
\hline$\omega\left[E J / \mu l^{4}\right]$ & $\sqrt{k^{4} \pi^{4}+k^{2} \pi^{2} \lambda_{\varphi}^{4}+\lambda_{w}^{4}}$ & $k^{2} \pi^{2}$ & $\frac{k^{2} \pi^{2}}{\sqrt{1+k^{2} \pi^{2} \zeta}}$ \\
\hline
\end{tabular}

On the basis of the formula (2.41), it is possible to determine the dependence that expresses the vibration frequency of the compressive force for the simply supported beam. For other conditions of the beam support, we can determine the value of natural frequencies and critical forces, and the relationship between them, from the following conditional equations:

- for the clamped-clamped beam:

$$
-2 A B(1-\cosh p \cos m)+\left(A^{2}-B^{2}\right) \sinh p \sin m=0
$$

- for the clamped-hinged beam:

$$
(A p-B m)[A \cosh p \sin m+B \sinh p \cos m]=0
$$

- for the cantilever beam:

$$
\begin{aligned}
& A B\left[\left(m^{2}-p^{2}\right)+\varepsilon^{2}(A p+B m)\right]-\left[p m\left(A^{2}-B^{2}\right)+A B \varepsilon^{2}(A p+B m)\right] \cosh p \cos m+ \\
& +A B\left[2 p m-\varepsilon^{2}(A m-B p)\right] \sinh p \sin m=0 .
\end{aligned}
$$

Equations (2.46)-(2.48) determine the dependence of the frequency of vibration of the compressive force for the assumed parameters of elastic foundation and the slenderness of the beam. 


\section{EXAMPLES AND DISCUSSIONS}

\subsection{EXAMPLE 1}
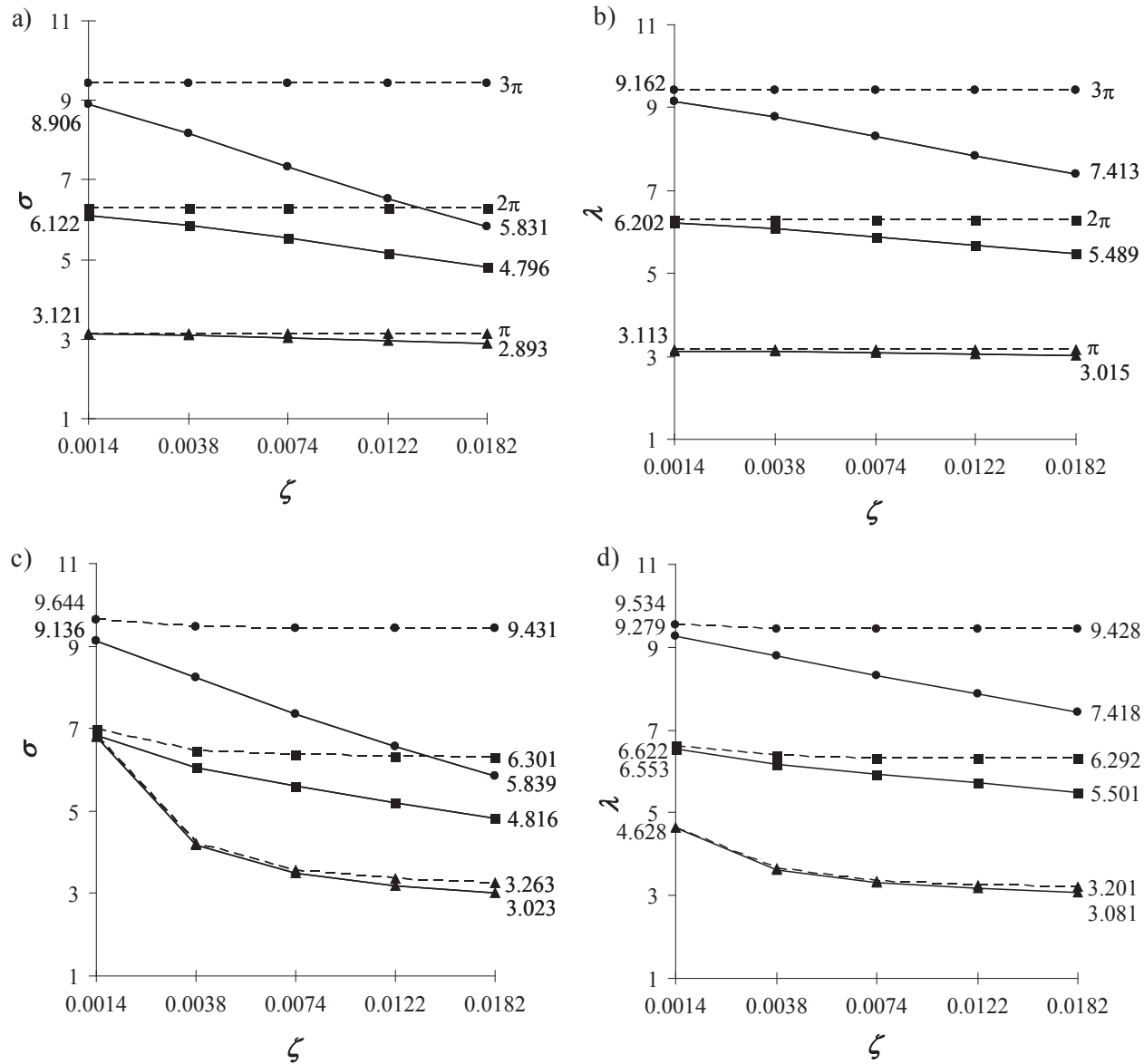

Fig. 4. Non-dimensional critical forces $\sigma$ (a) and non-dimensional frequencies of vibration $\lambda$ (b) for simply supported beam with no elastic foundations to rest on. Influence of elastic foundations on $\sigma$ (c) and on $\lambda$ (d); Bernoulli-Euler beam theory (---), Timoshenko beam theory $(-)$, first values $(\boldsymbol{\Lambda})$, second values $(\boldsymbol{\bullet})$, third values $(\bullet)$

A reinforced concrete simply supported beam of length $l=8 \mathrm{~m}$ resting on elastic foundation is considered. The beam with rectangular cross-section (the shear factor $\kappa=1.2)$ with width $b=0.5 \mathrm{~m}$ and beam cross-section height changing $(h=0.6 \div 2.2 \mathrm{~m})$ will be taken into account. The beam has Young's modulus $E=31 \times 10^{9} \mathrm{~Pa}$, the Poisson's ratio $v=0.2$ and the mass density $\rho=2500 \mathrm{kgm}^{-3}$. The moduli of foundation are $k_{w}=24.52 \times 10^{9} \mathrm{Nm}^{-2}$ and $k_{u}=5.88 \times 10^{6} \mathrm{Nm}^{-2}$. 
The effects of shear deformation on non-dimensional frequencies of vibration $\lambda$ and critical forces $\sigma$ are shown in Figure 4. For beams with large slenderness ratios (the smaller $\zeta$ ), the transverse shear has little effect. Thus, the Bernoulli-Euler beam theory can accurately predict the frequencies and critical forces. For beams with small slenderness ratios (the largest $\zeta$ ), the frequency and critical force are significantly smaller than predicted by the Bernoulli-Euler theory. For example, the first three frequencies are more than $4 \%, 14 \%$ and $27 \%$ lower than those given by Bernoulli-Euler theory for $\zeta=0.0182$ and in the cases of the first three critical forces are more than $8 \%, 31 \%$ and $61 \%$. It should also be noted that the influence of shear deformation on the next eigenvalues increases.
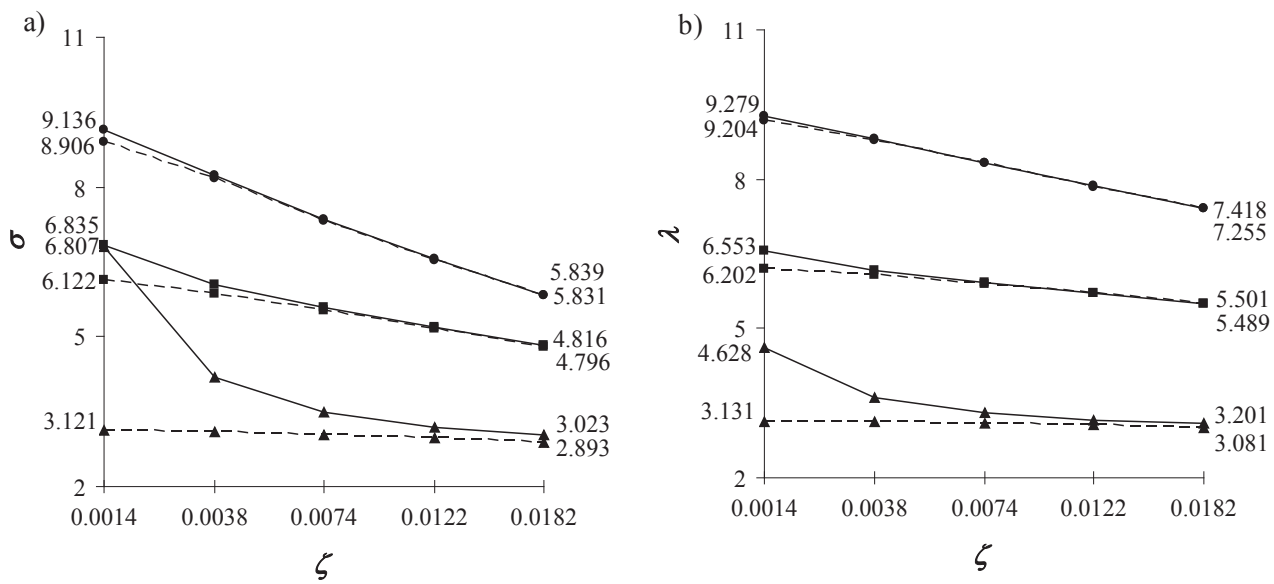

Fig. 5. Influence of elastic foundations on non-dimensional critical values $\sigma$ (a) and on non-dimensional frequencies of vibration $\lambda$ (b); values for beam with no elastic foundations to rest on (---), values for beam with elastic foundations to rest on $(-)$; first values $(\boldsymbol{\Delta})$, second values $(\boldsymbol{\bullet})$, third values $(\bullet)$

The effect of elastic foundations parameters on the critical values and frequencies of vibration are shown in Figure 5. The results show that the foundation parameters significantly affect values $\sigma$ and $\lambda$ for beams with large slenderness ratios (the smaller $\zeta$ ). For example, for $\zeta=0.0014$, the fundamental frequency and the first critical force are more than $32 \%$ and $54 \%$ higher than those given without considering the impact of elastic foundation. For $\zeta=0.0182$, differences in eigenvalues are $2 \%$ and $4 \%$. It can be seen that the impact is lower while determining the frequency of vibration. The influence of elastic foundation on the next eigenvalues decreases. 

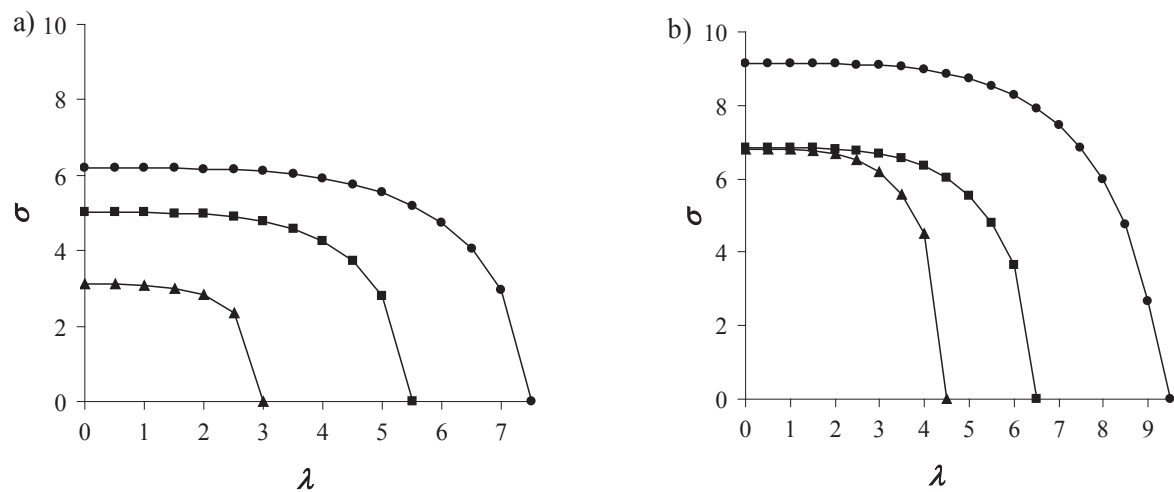

Fig. 6. The dependence between non-dimensional frequencies of vibration $\lambda$ and non-dimensional critical values $\sigma$ for $\zeta=0.0014$ (a) and for $\zeta=0.015$ (b)

It is possible to determine the dependence for vibration frequency of the compressive force for two cases $\zeta=0.0014$ (Figure 6a) and $\zeta=0.015$ (Figure 6b) on the basis of the formula (2.59). As the compressive force increases, the beam vibrations decrease and the critical value stops the beam vibrating. It is the dependence of bifurcation.
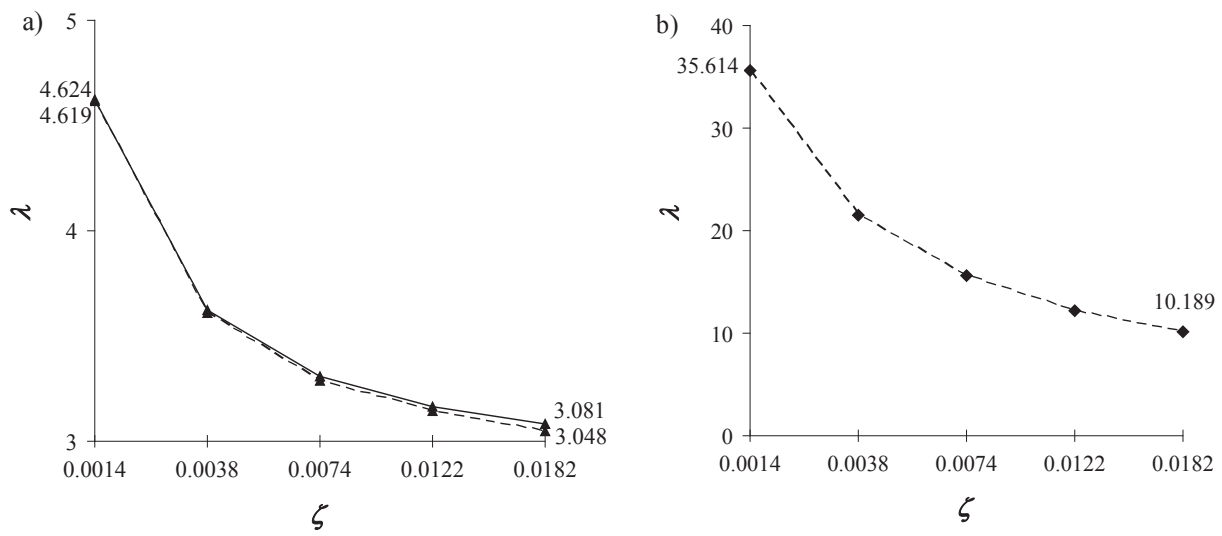

Fig. 7. Effect of rotational inertia on non-dimensional fundamental frequencies of vibration $\lambda$ : (a) first natural frequency band, (b) second natural frequency band; values for beam with effect of rotational inertia $(--)$, values for beam with no effect of rotational inertia $(-)$

The effect of rotational inertia on non-dimensional fundamental frequencies of vibration $\lambda$ is shown in Figure 7a. The effect is small, as for example, for $\zeta=0.0182$ the first frequency is about $1 \%$ lower than that given without considering the rotational inertia. But if the rotational inertia is accounted for, the second natural frequency band 
is received (Figure $7 \mathrm{~b}$ ). The influence of elastic foundation for this band is insignificant $(0.01 \%)$.

\subsection{EXAMPLE 2}

In the second example, the three exact frequency parameters of the beam are determined. Four kinds of end conditions, i.e. simply supported (Table 2), clamped- hinged (Table 3), clamped-clamped (Table 4) and cantilever beam (Table 5) are consider in this study. For the first case, solutions were calculated directly from the analytical closed form expression (2.43) ${ }_{1}$ and (2.45), for others - from equations (2.46)-(2.48), assuming $\sigma=0$. The beams resting on Winkler foundation $\left(\lambda_{w}\right)$, on two-parametrical foundation $\left(\lambda_{w}, \lambda_{\varphi}\right)$ and with no elastic foundation to rest on are considered.

The following mechanical and geometric properties of Timoshenko beam used by Yokoyama [26] are chosen for the analysis: the Poisson's ratio $v=0.25$, the shear coefficient: $\kappa=1.2$ (for rectangular cross-section), the slenderness ratio: $\sqrt{J / A l^{2}}=10$. Consequently, the shear deformation parameter is: $\zeta=0.03$. The foundation parameters $\lambda_{w}=0.88 \pi$ and $\lambda_{\varphi}=1$ are assumed.

For Bernoulli-Euler beam, frequency parameters were obtained assuming $\zeta=0$ (the effect of shear deformation was disregarded) and $n=0$ (the effect of rotatory inertia was disregarded). The results received in this way are known from literature. This proves the formulas developed in this paper are correct.

Table 2

Values of frequency parameter $\lambda$ for simply supported beam

\begin{tabular}{|c|c|c|c|c|}
\hline & & & Tim & \\
\hline & & -Euler & $\begin{array}{l}\text { without rotatory } \\
\text { inertia }\end{array}$ & $\begin{array}{c}\text { with rotatory } \\
\text { inertia }\end{array}$ \\
\hline $1 \mathrm{st}$ & without elastic foundation & 3.142 & 2.944 & 2.902 \\
\hline & Winkler foundation & 3.533 & 3.400 & 3.350 \\
\hline & two-parametrical foundation & 3.588 & 3.458 & 3.388 \\
\hline $2 \mathrm{nd}$ & without elastic foundation & 6.283 & 5.168 & 5.057 \\
\hline & Winkler foundation & 6.341 & 5.271 & 5.157 \\
\hline & two-parametrical foundation & 6.380 & 5.285 & 5.173 \\
\hline $3 \mathrm{rd}$ & without elastic foundation & 9.425 & 6.812 & 6.684 \\
\hline & Winkler foundation & 9.442 & 6.858 & 6.728 \\
\hline & two-parametrical foundation & 9.468 & 6.863 & 6.735 \\
\hline
\end{tabular}


Table 3

Values of frequency parameter $\lambda$ for clamped-hinged beam

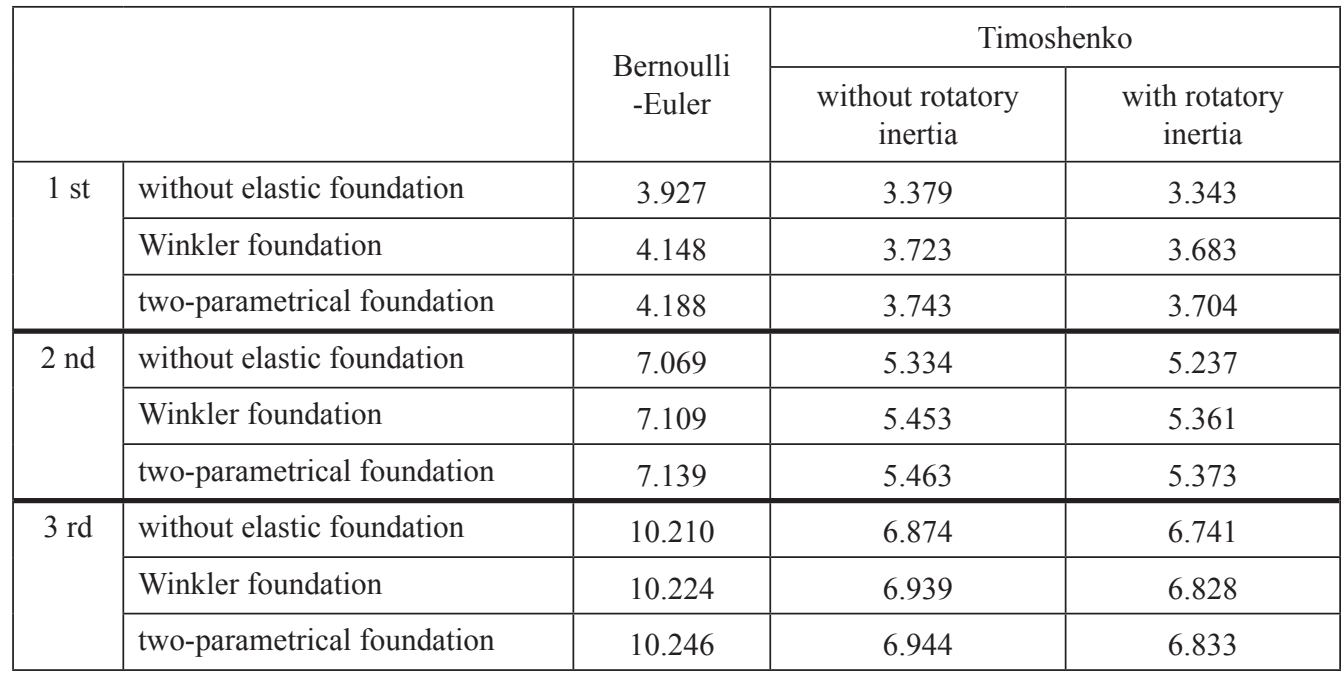

Table 4

Values of frequency parameter $\lambda$ for clamped-clamped beam

\begin{tabular}{|c|l|c|c|c|}
\hline \multicolumn{2}{|c|}{} & \multirow{2}{*}{$\begin{array}{c}\text { Bernoulli } \\
\text {-Euler }\end{array}$} & $\begin{array}{c}|c| \\
\text { without rotatory } \\
\text { inertia }\end{array}$ & $\begin{array}{c}\text { with rotatory } \\
\text { inertia }\end{array}$ \\
\cline { 3 - 5 } $1 \mathrm{st}$ & without elastic foundation & 4.730 & 3.759 & 3.741 \\
\cline { 2 - 5 } & Winkler foundation & 4.862 & 4.045 & 4.027 \\
\cline { 2 - 5 } & two-parametrical foundation & 4.889 & 4.051 & 4.034 \\
\hline \multirow{2}{*}{$2 \mathrm{nd}$} & without elastic foundation & 7.853 & 5.471 & 5.377 \\
\cline { 2 - 5 } & Winkler foundation & 7.883 & 5.605 & 5.522 \\
\cline { 2 - 5 } & two-parametrical foundation & 7.907 & 5.612 & 5.532 \\
\hline $3 \mathrm{rd}$ & without elastic foundation & 10.996 & 6.935 & 6.806 \\
\cline { 2 - 5 } & Winkler foundation & 11.007 & 7.018 & 6.950 \\
\cline { 2 - 5 } & two-parametrical foundation & 11.025 & 7.021 & 6.950 \\
\hline
\end{tabular}


Values of frequency parameter $\lambda$ for cantilever beam

\begin{tabular}{|c|c|c|c|c|}
\hline & & & Tim & \\
\hline & & -Euler & $\begin{array}{l}\text { without rotatory } \\
\text { inertia }\end{array}$ & $\begin{array}{l}\text { with rotatory } \\
\text { inertia }\end{array}$ \\
\hline $1 \mathrm{st}$ & without elastic foundation & 1.875 & 1.814 & 1.798 \\
\hline & Winkler foundation & 2.901 & 2.765 & 2.765 \\
\hline & two-parametrical foundation & 2.920 & 2.765 & 2.765 \\
\hline $2 \mathrm{nd}$ & without elastic foundation & 4.694 & 3.962 & 3.820 \\
\hline & Winkler foundation & 4.829 & 4.014 & 3.864 \\
\hline & two-parametrical foundation & 4.879 & 4.075 & 3.924 \\
\hline $3 \mathrm{rd}$ & without elastic foundation & 7.855 & 5.879 & 5.642 \\
\hline & Winkler foundation & 7.885 & 5.866 & 5.607 \\
\hline & two-parametrical foundation & 7.916 & 5.888 & 5.631 \\
\hline
\end{tabular}

For Timoshenko beams, the frequency parameters are significantly smaller than those obtained for the Bernoulli-Euler beams. The biggest differences are found for the case of clamped-clamped beam. The first three frequency are over $26 \%, 46 \%$ and $61 \%$ lower than those given for Bernoulli-Euler beam. This also proves that shear deformation and rotational inertia produce an increasing effect on the next values. It is very important the influence of rotational inertia on non-dimensional fundamental frequencies of vibration is small (of an order of $2 \%$ ). The biggest effect is produced for the hinged-hinged beam and the smallest for the clamped-clamped beam.

If the beams resting on elastic foundation are considered, the frequency parameters will be higher than those obtained for beams with no elastic foundation to rest on. The effect is the biggest for the hinged-hinged beam and the smallest for the clampedclamped beam. In the first case, the first frequency is over $17 \%$ higher than that given without considering the impact of elastic foundation and in the second $-8 \%$. The decreasing effect on the next frequencies is observed.

No matter what the conditions of the beam support are, the influence of the second foundation parameter, which is a function of the total rotation of the beam, is small.

\section{Conclusions}

The vibrations and stability of uniform beams resting on continuous two-parameter elastic foundation were studied. The equation of motion for Timoshenko and Bernoulli-Euler beam was derived. The relationships between the parameters describing vibration, the compressive force and the foundation parameters were investigated. 
Using analytical formulas developed in this paper, it was possible to obtain the non-dimensional parameters $\sigma$ and $\lambda$, which describe the critical force and vibration frequencies. The individual effect of foundation stiffness parameters, transverse shear deformation and rotatory inertia on eigenvalues of the beam can be examined by performing a parametric study.

\section{REFERENCES}

1. Bołotin, W.W.: Dynamic Stability of Elastic Systems, Moskwa, 1956. (in Russian).

2. Timoshenko, S.P., Gere, J.M.: Theory of elastic stability, McGraw-Hill, New York, 1961.

3. Wolmir, A.C.: Stability of Elastic Systems, Moskwa, 1963. (in Russian).

4. Timoshenko, S.P.: On the correction for shear of the differential equation for transverse vibrations of prismatic bars, Philosophical Magazine, Vol. 41, 744-746, 1921.

5. Timoshenko, S.P.: On the transverse vibrations of bars of uniform cross- section, Philosophical Magazine, Vol. 43, 125-131, 1922.

6. Gryczmański, M., Jurczyk, P.: The Subsoil Models and their Evaluation, Inżynieria i Budownictwo, Vol. 2, No. 95, 98-104, 1995. (in Polish).

7. Jemielita, G., Szcześniak, W.: Methods for Foundation Modeling, Prace Naukowe Politechniki Warszawskiej, Vol. 120, 1-33, 1993. (in Polish).

8. Winkler, E.: Die Lehre von der Elastizität und Festigkeit, Dominicus, Prague, (1867).

9. Thambiratnam, D., Zbuge, Y.: Free vibration analysis of beam on elastic foundation, Computers and Structures, Vol. 60, No. 6, 971-980, 1996.

10. Chen, C.N.: DQEM vibration analyses of non-prismatic shear deformable beams resting on elastic foundations, Journal of Sound and Vibration, Vol. 255, No. 5, 989-999, 2002.

11. De Rosa, M.A.: Stability and dynamics of beams on Winkler elastic foundation, Earthquake Engineering and Structural Dynamics, Vol. 18, 377-388, 1989.

12. Fargitaly, S.H., Zeid, K.M.: An exact frequency equation for an axially loaded beam-mass-spring system resting on a Winkler elastic foundation, Journal of Sound and Vibration, Vol. 185, No. 2, 357-363, 1995.

13. Nageswara, Rao B., Venkateswara, Rao G.: Post-critical behaviour of Euler and Beck columns resting on an elastic foundation, Journal of Sound and Vibration, Vol. 2764, 1150-1158, 200.

14. Song Xi, Soi- Rong Li.: Thermal buckling and post-buckling of pinned-fixed Euler-Bernoulli beams on an elastic foundation, Mechanics Research Communications, Vol. 34, 164-171, 2006.

15. Kim, S.M.: Vibration and stability of axial loaded beams on elastic foundation under moving harmonic loads, Engineering Structures, Vol. 26, 95-10, 20045.

16. Kim, S.M., Cho, Y. H.: Vibration and dynamic buckling of shear beam-columns on elastic foundation under moving harmonic loads, International Journal of Solids and Structures, Vol. 43, 393-412, 2006.

17. Sato, M., Kanie, S., Mikami, T.: Mathematical analogy of a beam on elastic supports as a beam on elastic foundation, Applied Mathematical Modelling, Vol. 32, 688-699, 2008.

18. Pasternak, P.Ł.: On a new method of analysis of an elastic foundation by means of two foundation constants, Gosstrojizdat, Moscow, 1954. (in Russian).

19. Filonienko - Borodich, M.M.: Some approximate theories of elastic foundation, Uchenyie Zapiski Moskovskogo Gosudarstvennogo Universtiteta, Mechanika, Vol. 46, 3-18, 1940. (in Russian).

20. Vlasow, V.Z., Loentiev, U.N.: Beams, plates and shells on elastic foundation, Gosfizmat, Moskow, 1966. (in Russian). 
21. Gomuliński, A.: Determination of eigenvalues for circular plates resting on elastic foundation with two moduli, Archives of Civil Engineering, Vol. XIII, No. 2, 183-203, 1967. (in Polish).

22. Ayvaz, Y., Daloglu, A.: Earthquake analysis of beams resting on elastic foundations by using a modified Vlasov model, Journal of Sound and Vibration, Vol. 200, No. 3, 315-325, 1997.

23. Ayvaz, Y.: Application of modified Vlasov model to free vibration analysis of beams resting on elastic foundations, Journal of Sound and Vibration, Vol. 255, No. 1, 111-127, 2002.

24. Naidu, N.R., Rao, G.V.: Stability behaviour of uniform beams on a class of two-parameter elastic foundation, Computers and Structures, Vol. 57, No. 3, 551-553, 1995.

25. Naidu, N.R., Rao, G.V.: Vibrations of initially stressed uniform beams on two-parameter elastic foundation, Computers and Structures, Vol. 57, No. 2, 941-943, 1995.

26. Yokoyama, T.: Vibration analysis of Timoshenko beam-columns on two-parameter elastic foundations, Computers and Structures, Vol. 61, No. 6, 995-1007, 1995.

27. El - Mously, M.: Foundamental frequencies of Timoshenko beams mounted on Pasternak foundation, Journal of Sound and Vibration, Vol. 228, No. 2, 452-157, 1999.

28. Arboleda- Monsalve, L.G., Apata- Medina, D.G., Aristizabal - Ochoa, J.D.: Timoshenko beamcolumn with generalized and conditions on elastic foundation: Dynamic-stiffness matrix and load vector, Journal of Sound and Vibration, Vol. 310, 1057-1079, 2008.

29. De Rosa, M.A.: Free vibrations of Timoshenko beams on two-parameter elastic foundation, Computers and Structures, Vol. 57, No. 1, 151-156, 1995.

30. Filipich, C.P., Rosales, M.B.: A further study about the behaviour of foundation piles and beams in a Winkler-Pasternak soil, International Journal of Mechanical Sciences, Vol. 44, 21-36, 2002.

31. Chen, W.Q., Lü, C.F., Bian, Z.G.: A mixed method for bending and free vibration of beams resting on a Pasternak elastic foundation, Applied Mathematical Modelling, Vol. 28, 877-890, 2004.

32. Matsunaga, H.: Vibration and buckling of deep beam-columns on two-parameter elastic foundations, Journal of Sound and Vibration, Vol. 228, No. 2, 359-376, 1999.

33. Ying, C.F. Lü, Chen, W.Q.: Two-dimensional elasticity solutions for functionally graded beams resting on elastic foundations, Composite Structures, Vol. 84, 209-219, 2008. 